\title{
A psychophysiological influence on the choice of illicit drugs
}

\author{
EDWARD DEAUX \\ Polydrug Research Unit, Philadelphia Psychiatric Center, Philadelphia, Pennsylvania 19004 \\ and \\ Antioch College, Yellow Springs, Ohio 45387
}

\begin{abstract}
The variable of "augmenting" and "reducing," as measured by kinesthetic figural aftereffects, was found to relate to the choice of amphetamines or barbiturates as a predominant drug, and differences were also found between males and females within each drug category. At the conceptual level, the need for stimulation ("nStim") is presented as a hypothetical construct which relates the KFA measure to the choice of drug. The strength of the relationship and the significance of the results suggest the possible use of the KFA test in determining the appropriateness of prevention and treatment methods, which are typically applied without the specific consideration of the need for stimulation.
\end{abstract}

On the basis of their studies on individual differences in response to sensory stimulation, Buchsbaum and his associates have proposed a model of a central nervous system (CNS) mechanism which modulates the intensity of sensory input (Buchsbaum \& Pfefferbaum, 1972; Buchsbaum \& Silverman, 1968; Landau, Buchsbaum, Carpenter, Strauss, \& Sacks, 1975). The endpoints of the continuum of stimulus intensity control are represented by the "augmenter," who tends to increase the perceived intensity of stimuli, and the "reducer," who tends to decrease it. These labels were originally of fered by Petrie (1967), who differentiated between augmenters and reducers on the basis of kinesthetic figural aftereffects (KFA), a phenomenon which lends itself to rapid and inexpensive measurement.

The typical procedure for measuring KFA is simple: The subject, who is blindfolded throughout the session, is given two wooden blocks, one for each hand. The preferred hand feels the width of a standard block, and, with the other hand, the subject finds the point on a tapered block that is the same width, subjectively, as the standard. After several baseline judgments, the subject is given a larger "stimulation" block for his preferred hand to rub while the other hand rests. This stimulation period is immediately followed by another series of judgments with the standard block, and the stimulation-measurement series is repeated for two additional times. The change from the mean of the prestimulation baseline judgments to the mean of the poststimulation judgments is taken as the KFA score. According to Petrie's ter-

This research was supported by Grant H81 DA01657 from the National Institute on Drug Abuse, Dr. John Benvenuto and George Beschner, Project Officers. The Project Director was Dr. Alfred S. Friedman, Director of Research, Philadelphia Psychiatric Center. The author's mailing address is: Polydrug Research Center, 301 City Line Avenue, Bala Cynwyd, Pennsylvania 19004. minology, "augmenters" tend to increase their poststimulation judgments relative to baseline, whereas "reducers" decrease their judgments after stimulation with the larger block.

A number of individual tendencies and characteristics have been found to be associated with the KFA measure, and they have generally been attributed to an underlying "stimulus intensity" or "strength" variable. Briefly, since reducers are essentially damping stimulus input, they tend to have high pain tolerance (Petrie, 1960) but low tolerance for stimulus deprivation situations (Petrie, Collins, \& Solomon, 1958) relative to augmenters, who heighten input. Further, the amplitude of electroencephalographic visual evoked responses has been found to correlate significantly with KFA scores (Spilker \& Callaway, 1969), with reducers exhibiting a diminished response at higher input intensities, substantiating the physiological substrate of the augmentingreducing cortical variable.

The possibility of this variable's being related to drug choice was raised by Gupta (1974), who explored the influence of specific drugs on the KFA. In an extensive design, dexedrine $(10 \mathrm{mg})$, phenobarbital (100 mg), and a placebo were administered orally to subjects over a number of sessions, in each of which KFA was measured, so that each subject acted as his own control. The results were clear and significant: Dexedrine produced relative augmentation, and phenobarbital led to reduction, relative to the placebo control measurements. From the conjunction of the facts that (a) individuals differ markedly on the KFA measure and (b) stimulants and depressants modify the KFA, and presumably the underlying stimulus intensity modulation, in a highly predictable direction, the following experimental hypothesis emerged: In the illicit use of stimulants and depressants, an individual's choice of primary drug 
is influenced by his or her position on the augmentingreducing variable. By comparing the results of the KFA test with the drug-use patterns of volunteer subjects who were participating in a survey of illicit polydrug use, it was possible to test this hypothesis.

Drug-use information reported here comes from the self report of the subjects. Although some feel that self-report data lack a certain degree of believability, if not validity; there were a number of factors functioning in the present study which warrant its use. First, the subjects were paid volunteers, and, once accepted as subjects, they received $\$ 20$ regardless of the information they provided: Thus, they had no motive for reporting false drug-use data. Second, the staff members who conducted the 6-h interview and testing session were consistently and pointedly nonjudgmental in their approach to the subjects' use of drugs. Finally, since the interest of the present study was to assign subjects to two drugpattern groups on the basis of comparatively gross measures of drug use, when a subject could not be assigned readily to the barbiturate-using or amphetamine-using group, that subject was excluded from this study.

\section{METHOD}

\section{Subjects}

On the basis of lengthy and extensive drug histories and drug-use questionnaires, the subjects who qualified for inclusion in this study were assigned to one of four cells of a 2 by 2 factorial design defined by the variables of sex and the drug of predominant use (barbiturates vs. amphetamines). In the course of interviewing a total of 366 subjects, the first 12 subjects who were assigned to each cell determined that cell's composition. The mean age of the 24 males included in the study was 21.9 years; the mean age of the females was 21.8 years.

The determination of the predominant drug of use (or abuse) was made as follows: If either a barbiturate or an amphetamine was a subject's first or second most frequently used drug, by choice, and the other (that is, amphetamine or barbiturate, respectively) did not appear within the next five most frequently used drugs, the subject was classified as a barbiturate or amphetamine user, depending on which was most frequently used. A complete drug screen was conducted on a sample of each subject's urine by thin-layer chromatography, and no one was found to have any drug present at the time of testing.

\section{Procedure}

Upon assignment to a cell in the study, each subject was taken to an isolated room, blindfolded, and given the KFA test. The test is an abbreviated version of the procedure detailed by Petrie (1967), which entailed two sets of stimulation and comparison blocks. Gupta (1974), Sales (1972), and others employed only the larger stimulation block, and, due to time constraints, the larger set was also used here. Determination of the subject's preferred hand was followed by the presentation of the standard, $3.81-\mathrm{cm}(1.5$-in. $)$ block to that hand and a tapered block to the nonpreferred hand. The tapered block was $76.20 \mathrm{~cm}$ (30 in.) in length; its width increased from $1.27 \mathrm{~cm}(0.5 \mathrm{in}$.$) at the end nearest the subject to 10.16 \mathrm{~cm}$ (4.0 in.) at the other end. Following two practice trials, the subject was asked to find the point on the tapered block which was the same subjective width as the standard block in the preferred hand. The determination was made throughout by ascending trials; in other words, the subject moved his or her nonpreferred hand from the smaller end of the tapered block toward the wider end, stopping at the point of subjective equality. Between judgment trials, the subject's hand was returned to the smaller end of the tapered block. The subject's measurements were made only by the thumb and forefinger, which were held in a small bracket that slid along a rod mounted above the tapered block and attached at each end. A pointer on the sliding bracket aliowed precise determination of the subject's choice.

After the initial series of four measurements, the subject was given a larger, $6.35-\mathrm{cm}(2.5$-in.) block to rub between the thumb and forefinger of his or her preferred hand, to provide "stimulation" between the test trials. The first period of stimulation lasted $90 \mathrm{sec}$, after which another series of four judgments was made. The second stimulation period followed immediately after the second series of trials and lasted for another $90 \mathrm{sec}$. The third judgment series was followed by $120 \mathrm{sec}$ of stimulation with the larger block, after which the final four judgments were made. During all stimulation periods, the subject's nonpreferred hand rested in a comfortable position with the fingers free and not touching anything.

It should be noted that although it was obvious to the subjects that the block they were given to rub (for the stimulation period) was different from the block that they felt during their judgment trials, no instruction implied that the original standard block was returned to them on poststimulation measuring trials. Thus there was no indication given that the subject was measuring the same standard block following each stimulation period.

\section{RESULTS}

Two KFA scores for each subject were considered in the analysis of the results. The first is the baseline measure, which indicates the subject's initial, prestimulation subjective judgment of the width of the standard block. The second score is the change that was found between the mean of the prestimulation baseline measurements and the mean of the judgments made after the stimulation periods with the large block.

The mean and standard error for each of the two scores are presented for the four groups separately in Table 1. The baseline scores represent the distance from the smaller end of the tapered block to the point of subjective equality, measured in linear centimeters. The point at which the tapered block is $3.81 \mathrm{~cm}$ in width, and therefore the same physical width as the standard block used in the judgments, is $21.77 \mathrm{~cm}$ from the block's smaller end. The average judgment in each of the four groups is below this point: The mean of the barbiturate-using group is $16.84 \mathrm{~cm}$, whereas the amphetamine-using group's mean is $19.74 \mathrm{~cm}$, an average difference of $2.9 \mathrm{~cm}$. Also, within each of the drug-use categories, the mean baseline score for the males is higher. An analysis of variance was conducted on these data, and the results of that analysis are presented in the left part of Table 2. The between-group difference attributable to the drug category is significant $(p<.05)$, but the difference between sexes is not. Likewise, there is no apparent interaction in the baseline measure between the drug type and the subject's sex.

The change scores, also presented in Table 1, represent the increase $(+)$ or decrease $(-)$ in the subject's point of subjective equality from the mean of the 
Table 1

Results of the Kinesthetic Figural Aftereffects Test

\begin{tabular}{lcccc}
\hline \multicolumn{2}{c}{ Baseline $(\mathrm{cm})$} & \multicolumn{2}{c}{ Change Score $(\mathrm{cm})$} \\
\multicolumn{1}{c}{ Group } & Mean & $( \pm$ S.E. $)$ & Mean & $( \pm$ S.E. $)$ \\
\hline $\begin{array}{l}\text { Barbiturate } \\
\text { Males }\end{array}$ & 17.437 & $( \pm 1.299)$ & -1.133 & $( \pm .795)$ \\
$\begin{array}{l}\text { Females } \\
\text { Amphetamine }\end{array}$ & 16.252 & $( \pm .755)$ & +1.034 & $( \pm .626)$ \\
$\begin{array}{l}\text { Males } \\
\text { Females }\end{array}$ & 20.420 & $( \pm 1.277)$ & -5.217 & $( \pm .740)$ \\
\hline
\end{tabular}

Table 2

Statistical Analyses Conducted on the KFA Data

\begin{tabular}{|c|c|c|c|c|c|c|}
\hline \multirow[b]{2}{*}{ Source } & \multicolumn{3}{|c|}{$\begin{array}{c}\text { Baseline } \\
\text { Analysis of Variance }\end{array}$} & \multicolumn{3}{|c|}{$\begin{array}{c}\text { Change Score } \\
\text { Analysis of Covariance }\end{array}$} \\
\hline & df & M.S. & $\mathrm{F}$ & df & M.S. & $\mathrm{F}$ \\
\hline Drug & 1 & 15.474 & $6.634^{*}$ & 1 & 12.157 & $25.145 * *$ \\
\hline Sex & 1 & 3.064 & 1.313 & 1 & 4.992 & $10.326 * *$ \\
\hline Drug by Sex & 1 & .018 & .008 & 1 & .001 & .017 \\
\hline Error & 44 & 2.332 & & 43 & .484 & \\
\hline
\end{tabular}

baseline judgments to the mean of the judgments which followed stimulation with the larger block. Again, systematic differences between groups are apparent: The mean of the barbiturate-using subjects is $-0.05 \mathrm{~cm}$, and the amphetamine-using group's mean is $-4.06 \mathrm{~cm}$, a difference which is considerably larger than that found in the baseline measures. Within the barbiturate groups, the mean differerice between males and females is $2.16 \mathrm{~cm}$, and it should be noted that females increased their poststimulation judgments while males lowered theirs. A similar difference was found among amphetamine users; although both subgroups decreased their judgments after the stimulation periods, males did so $2.29 \mathrm{~cm}$, on the average, more than females.

Due to the differences in the baseline measure, an analysis of covariance (Winer, 1962) was conducted on the change scores, using the baseline scores as the covariate measure. The results of this analysis are presented in the right part of Table 2 , where it can be seen that the differences due to the drug of predominant use and to sex are both highly significant $(p<.001)$. As with the baseline measure, however, there is no interaction between the two variables.

To briefly summarize the results, barbiturate users and amphetamine users were found to differ significantly on both their initial, prestimulation judgments of the width of the standard $3.81-\mathrm{cm}$ block and the changes that occurred in their size judgments following the stimulation with the $6.35-\mathrm{cm}$ block, which is the usual KFA measure. Only the group composed of female barbiturate users showed an increase from the pre- to the poststimulation series, and considerably greater reduction in this measure was found among amphetamine users. Furthermore, within each of the two drug-use categories, males reduced more than females, a difference which was also found to be significant.

\section{DISCUSSION}

The results provide strong support for the experimental hypothesis: Individuals whose predominant drug is a barbiturate were found to differ significantly on the KFA augmenting-reducing variable from individuals whose predominant drug is an amphetamine. Before considering further the differences between the drug groups, several secondary findings should be addressed. First, the overall mean baseline measure was considerably below the point of the physical equality. This "error" has been shown to be a function of the technique employed in making the size judgments; Wertheimer (1954a) established that the width of a block held between the thumb and forefinger of the preferred, or dominant, hand is judged to be smaller than a block of equal width held in the nonpreferred hand. This effect, which he named the "bilateral kinaesthetic difference" (BKD), has also been found to be significantly greater in females than in males (Wertheimer, 1954a), and a similar sex difference was found here, although it was not significant. Likewise, the sex difference in the KFA change score, which represents the influence of aftereffects, has precedence, not only for kinesthetic figural aftereffects (McEwen \& Rodger, 1960; Platt, Holzman, \& Larson, 1971; Wertheimer, 1954a), but for visual figural aftereffects as well (McEwen \& Rodger, 1960; Wertheimer, 1954b). These differences, therefore, have been found to obtain among the general population of subjects and are not characteristics specific to a sample of illicit drug users. The attention can once again return to the differences between the drug-use groups, with only a small preparatory caveat. Since three of the four groups' mean KFA change scores were in the reducing category, it is important to state that the terms "augmenting" and "reducing" are used here in a relative sense. For example, the statement that among males the barbiturate-using group was found to be relatively more augmenting than the amphetamine-using group does not carry the same meaning as it would if the barbiturate-using males had been found to be "augmenters" in the categorical use of the term. The KFA change score is employed in this study as a continuous measure, and the between-group differences are discussed within the context, and the distribution, of the total sample of 48 subjects.

The difference between the barbiturate-using group and the amphetamine-using group has been presented. A consideration of the direction of that difference is necessary before the inference can be made that the internal variable underlying the KFA measure influences the choice of drugs. As mentioned earlier, 
Gupta (1974) found that, in a sample of non-drug-using subjects, a barbiturate produced relative reduction on the KFA whereas an amphetamine produced relative augmentation. The present study found barbiturate users to be more augmenting than amphetamine users, who were much more reducing. The symmetry of the two sets of findings is striking, and an underlying motivational variable has been proposed, at the conceptual level, which can explain not only the results but also their symmetry. The need for stimulation, nStim, has been defined as a basic motive, and the relationship between nStim and the KFA measure has been offered by Sales (1972) and Sales, Guydosh, and Iacono (1974). Individuals who are found to be reducers on the KFA are considered to have a relatively greater need for stimulation, and they seek to heighten stimulation through contact sports (Ryan \& Foster, 1967) and other activities that involve them in stimulating situations (Sales, 1972). Amphetamine use, previously shown to produce augmentation, may be thought of as a chemical means of increasing stimulation, and it follows that amphetamine users would be relatively more reducing, which is the finding of the present study. In the context of nStim, it can be stated that individuals who reduce have a greater need for stimulation, and one way in which some reducing individuals satisfy that need is through the use of amphetamines.

The current findings offer evidence for the converse relationship as well: Compared to amphetamine users, individuals who use barbiturates were found to be relative augmenters. Hence, not only may reducers be seeking to heighten stimulation through the use of amphetamines, but relative augmenters may be motivated to reduce stimulation and may choose the use of barbiturates to attain that goal. Conceptually, this implies that nStim, as a source of motivation, is homeostatic in nature. Thus, individuals who internally reduce stimulation seek to increase it, and those who internally augment stimulation are motivated to reduce it. Both types may choose to reach their sought after, and presumably mesial, level of stimulation through chemical means.

A question which arises from the present data is whether an individual's position on the augmentingreducing variable (or nStim) precedes (i.e., leads to) or follows (i.e., results from) the use of barbiturates or amphetamines as a predominant drug. In previous KFA research it has been assumed that the need for stimulation is a more or less stable motivational characteristic which acts to direct an individual toward activities that satisfy the need. For instance, the relationship between reduction and involvement in contact sports is presented in the context of reducers seeking stimulating activities rather than involvement in stimulating activities leading to internal reduction, as an adaptive or compensating response. Likewise, in the present report, it is hypothesized that one's being a relative reducer precedes his use of a stimulating drug. However, there is no definite evidence in the present study either to support or to reject that hypothesis. The data can provide only a suggestive answer to that question, and the answer is equivocal.

Were the KFA change score an indication of an adaptive response to, or a "rebound" effect from, the use of a stimulant or a depressant, one would expect to find a positive correlation between the length of time subjects used barbiturates and their KFA change score (which is relatively augmenting within this group) and, conversely, a negative correlation between duration of use and the KFA score among amphetamine users. To test this hypothesis, correlation coefficients were calculated for each drug-use group between the number of years each subject had used his or her predominant drug and the KFA change score. The within-group results reveal a tendency, although it is not significant, toward correlations in the direction opposite to that predicted by the adaptiveresponse hypothesis. For the barbiturate-using group, $\mathrm{r}=-.340$, and for the amphetamine-using group, $\mathrm{r}=+.094(\mathrm{df}=23$, critical value of $\mathrm{r}$ for $\mathrm{p}<.05$ is .396); the difference between the correlation coefficients is likewise nonsignificant $(z=1.45, p=$ $.074)$. This test, which is neither powerful nor precise, does not warrant the rejection of the adaptive-response hypothesis, but if it is true that the KFA indicates a reaction to the extended use of stimulants or depressants, that effect is not evident in the data. Further research may be able to provide a more definite answer to this question.

Unfortunately, no discussion of the KFA would be complete without some mention of the studies which have been published in recent years claiming to expose the instability of the KFA measure. Weintraub, Green, and Herzog (1973) presented an example of this type of report. However, the KFA measure has been shown to relate significantly and repeatedly with a number of behavioral, physiological, and personality variables. Furthermore, when the KFA test is used to assess differences between groups of individuals, as it was in the present study, or to determine the effects of specific drugs, as it was in Gupta's (1974) work, many of the problems attributed to the test are avoided.

Although inferential evidence is provided here for a conceptual variable which has been posited as the "source" of the KFA measure, and suggestions of an underlying psychophysiological, CNS mechanism have been made, the use of the KFA test does not require the acceptance of these concepts. It is important that (a) the KFA measure, as the data indicate, is significantly related to drug-use patterns which include barbiturates and amphetamines as predominant drugs, (b) it is determined quickly and inexpensively, and (c) it may be valuable as a diagnos- 
tic tool in determining prevention and/or treatment appropriateness. With regard to the last point, an extreme reducer who uses amphetamines and an augmenter who uses barbiturates may be expected to benefit differentially from any one prevention or treatment program, and the extent to which that program enhances or damps stimulation could be an important variable influencing the success or failure of the approach. Relaxation therapy or meditation, as examples, could conceivably, and inadvertently, encourage an extreme reducer to seek greater stimulation and create a situation in which the use of amphetamines is more highly motivated than it was before "treatment" began.

\section{REFERENCES}

Buchsbaum, M., \& Pfefferbaum, A. Individual differences in stimulus intensity response. Psychophysiology, 1972, 8, 600-611.

Buchsbaum, M., \& Silverman, J. Stimulus intensity control and the cortical response. Psychosomatic Medicine, 1968, 30, 12-22.

Gi'PTA. B. S. Stimulant and depressant drugs on kinaesthetic tigural aftereffects. Psychopharmacologia (Berlin), 1974, 36. $275-280$

Landau, S. G., Buchsbaum, M. S., Carpenter, W., Strauss, J., \& SACKs, M. Schizophrenia and stimulus intensity control. Archives of General Psychiatry, 1975, 32, 1239-1245.

McEwen, P., \& Rodger, S. Some individual differences in figural aftereffects. British Journal of Psychology, 1960, 51, 1-8.

PETRIE, A. Some psychological aspects of pain and the relief of suffering. Annals of the New York Academy of Science, 1960, 86, $12-27$.

Petrie, A. Individuality in pain and suffering. Chicago: University of Chicago Press, 1967.
Petrie, A., Collins, W., \& Solomon, P. Pain sensitivity, sensory deprivation and susceptibility to satiation. Science, 1958, 128. 1431-1433.

Platt, D.. Holzman. P. S.. \& Larson. D. Individual consistencies in kinesthetic figural aftereffects. Perceptual and Motor Skills, 1971, 32, 787-795.

Ryan, E. D., \& Foster, R. Athletic participation and perceptual augmentation and reduction. Journal of Personality and Social Psychology, 1967, 6, 472-476.

SAlES, S. M. Need for stimulation as a factor in preferences for different stimuli. Journal of Personality Assessment, 1972, 36, 55-61.

Sales, S. M.. Guydosh. R. M., \& Iacono. W. Relationship between strength of the nervous system and the need for stimulation. Journal of Personality and Social Psychology, 1974, 29. 16-22.

Spilker, B., \& Callaway, E. Augmenting and reducing in averaged visual evoked responses to sine wave light. Psychophysiology, 1969, 6, 49-57.

Weintraub, D. J., Green, G. S., \& Herzog, T. R. Kinesthetic aftereffects day by day: Trends, task features, reliable individual differences. American Journal of Psychology, 1973, 86, 827-844.

Wertheimer, M. Constant errors in the measurement of figural after-effects. American Journal of Psychology, 1954, 67. 543-546. (a)

WeRtheimer, M. The differential satiability of schizophrenic and normal subjects: A test of a deduction from the theory of figural after-effects. Journal of General Psychology. 1954. 51. 291-299. (b)

WINER, B. J. Statistical principles in experimental design. New York: McGraw-Hill, 1962. Pp. 781-792.

(Received for publication March 25, 1976; revision accepted May 5, 1976.) 